\title{
Guilty by Association: Platelet Reactivity and Inflammation in Psoriatic Arthritis
}

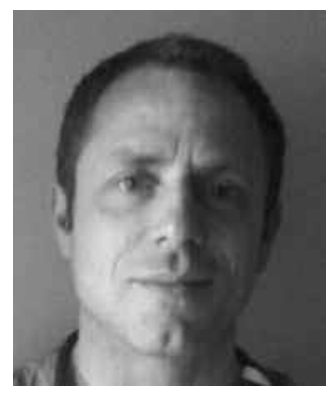

Patients with rheumatoid arthritis (RA) die prematurely from cardiovascular disease (CVD) ${ }^{1}$. The risk of adverse cardiovascular events and subsequent mortality in patients with RA is comparable to that associated with type 2 diabetes mellitus ${ }^{2}$. There is an emerging body of evidence that patients with other forms of inflammatory arthritis (IA), in particular psoriatic arthritis (PsA), are at a similarly elevated risk of $\mathrm{CVD}^{3}$. Platelets play a key role in the complex chain of events leading to the thrombotic occlusion of a coronary artery ${ }^{4}$. Antiplatelet therapy with aspirin and/or a variety of other agents is effective at reducing the mortality associated with incident $\mathrm{CVD}^{5}$ and is recommended for the primary prevention of same in patients at high risk of atherothrombotic events ${ }^{4}$. Despite the greatly increased risk of heart disease and strokes, there are currently no antiplatelet guidelines for the primary prevention of CVD in patients with $\mathrm{IA}^{3}$, and platelet function remains largely uninvestigated in this patient population. It is within this context that the recent work of Di Minno and colleagues, reported in this issue of The Journal, is both timely, and of high clinical importance ${ }^{6}$.

Platelet reactivity is a measure of how easily platelets will adhere to one another and aggregate to form thrombus. Several lines of evidence suggest that increased platelet reactivity is predictive of recurrent events in patients with established $\operatorname{CVD}^{7,8}$. Furthermore, recent work clearly demonstrates a definite link between increased platelet reactivity and heightened thrombogenicity in cardiovascular patients who demonstrate objective evidence of inflammation ${ }^{9}$. To date, the influence of inflammation on platelet reactivity in patients with PsA has not been evaluated.

Therefore, the study by Di Minno, et al examining platelet function in a significant number of patients with PsA, stratified according to disease activity and compared to age- and sex-matched controls, makes a solid contribution to this area and demonstrates a number of key findings. First, patients with PsA whose disease has been well controlled over a sustained period essentially have normal platelet function. Second, PsA patients who have objective evidence of active inflammation have an enhanced platelet response to both arachidonic acid and adenosine diphosphate (ADP), compared to healthy controls and those patients with PsA who are effectively in remission. This effect is independent of medication use, including biologic therapy, as all of the patients were receiving tumor necrosis factor- $\alpha$ blockers. Finally, C-reactive protein (CRP) levels correlate positively with platelet aggregation responses across the entire cohort, and the achievement of minimal disease activity is negatively associated with increased platelet reactivity in all the assays that were used. These findings are clear and convincingly shown, and should be of interest to rheumatologists and the wider medical community.

However, the study by Di Minno, et al also has some limitations. The platelet count is not reported, and by implication the results were not corrected for the possible influence of the well described reactive thrombocytosis that is associated with disease activity in PsA, although previous work has shown that platelet count does not significantly influence platelet aggregation measurements ${ }^{10}$. Also, the platelet function assays that were used assessed the platelet responses, separately, to each of only 2 concentrations of arachidonic acid and a single concentration of ADP, the concentrations tested were in the low submaximal range, and the clinical applicability of such an approach has been called into question ${ }^{11}$.

Platelet aggregation in vivo is complex and involves multiple agonists, receptor-effector mechanisms, and signaling pathways ${ }^{4}$. In a recent study of a similar number of patients with a variety of inflammatory arthritides, we used an assay that measures the response of platelets to multiple concentrations of multiple agonists, simultaneously, and found that patients with IA who are inflamed demonstrate increased platelet reactivity that is unique to the ADP pathway $^{12}$. A possible reason for the discordant finding with regard to the platelet responses to arachidonic acid between our work and that of Di Minno, et al may be that approximately $50 \%$ of our cohort were using nonsteroidal antiinflammatory drugs, which are known to affect this path-

See Platelet reactivity and disease activity in PsA, page 334 
way $^{13}$, whereas such patients were excluded from the current study. However, the consistent finding of an enhanced platelet response to ADP in IA and PsA patients with active disease is noteworthy. Numerous studies have shown a clear association between markers of inflammation such as CRP and a poor response to ADP blockade with clopidogrel in patients with CVD, and that a poor response to ADP inhibition predicts future adverse cardiovascular events ${ }^{9,14}$. Given the clear evidence that patients with IA are at greatly increased risk of $\mathrm{CVD}^{3}$, this platelet hyperreactivity in response to ADP in patients with PsA whose disease activity is poorly controlled may represent a potential prothrombotic bias and is certainly worthy of further investigation.

Finally, Di Minno and colleagues tested platelet reactivity on only one occasion across the entire cohort. To truly test the hypothesis that inflammation per se adversely increases platelet reactivity in IA/PsA and that improved disease control leads to a normalization of platelet function, sequential testing of platelet aggregation responses to several concentrations of a variety of agonists before and after the induction of remission in a previously inflamed group of patients is required. Such studies are currently lacking. Furthermore, it is not known if the use of specific antiplatelet agents targeting either the arachidonic acid pathway or the ADP-receptor in patients with IA/PsA can reduce the cardiovascular risk in this patient population, and further studies in this area are warranted.

PAUL MacMULLAN, MB, BCh, BAO, MRCPI, Department of Molecular and Cellular Therapeutics, Royal College of Surgeons in Ireland, 123 , St Stephen's Green, Dublin, 2, Ireland

Address correspondence to Dr. MacMullan;

E-mail:pmacmullan@hotmail.com

\section{REFERENCES}

1. Naranjo A, Sokka T, Descalzo MA, Calvo-Alen J, Horslev-Petersen $\mathrm{K}$, Luukkainen RK, et al. Cardiovascular disease in patients with rheumatoid arthritis: Results from the QUEST-RA study. Arthritis Res Ther 2008;10:R30.
2. van Halm VP, Peters MJ, Voskuyl AE, Boers M, Lems WF, Visser $\mathrm{M}$, et al. Rheumatoid arthritis versus diabetes as a risk factor for cardiovascular disease: A cross-sectional study, the CARRE Investigation. Ann Rheum Dis 2009;68:1395-400.

3. Peters MJ, Symmons DP, McCarey D, Dijkmans BA, Nicola P, Kvien TK, et al. EULAR evidence-based recommendations for cardiovascular risk management in patients with rheumatoid arthritis and other forms of inflammatory arthritis. Ann Rheum Dis 2010;69:325-31.

4. Davi G, Patrono C. Platelet activation and atherothrombosis. N Engl J Med 2007;357:2482-94.

5. Barrett NE, Holbrook L, Jones S, Kaiser WJ, Moraes LA, Rana R, et al. Future innovations in anti-platelet therapies. Br J Pharmacol 2008;154:918-39.

6. Di Minno MND, Iervolino S, Peluso R, Scarpa R, Di Minno G. Platelet reactivity and disease activity in subjects with psoriatic arthritis. J Rheumatol 2012;39:334-6.

7. Harrison P, Keeling D. Platelet hyperactivity and risk of recurrent thrombosis. J Thromb Haemost 2006;4:2544-6.

8. Angiolillo DJ, Bernardo E, Sabate M, Jimenez-Quevedo P, Costa MA, Palazuelos $\mathrm{J}$, et al. Impact of platelet reactivity on cardiovascular outcomes in patients with type 2 diabetes mellitus and coronary artery disease. J Am Coll Cardiol 2007;50:1541-7.

9. Gurbel PA, Bliden KP, Kreutz RP, Dichiara J, Antonino MJ, Tantry US. The link between heightened thrombogenicity and inflammation: Pre-procedure characterization of the patient at high risk for recurrent events after stenting. Platelets 2009;20:97-104.

10. Mani H, Luxembourg B, Klaffling C, Erbe M, Lindhoff-Last E. Use of native or platelet count adjusted platelet rich plasma for platelet aggregation measurements. J Clin Pathol 2005;58:747-50.

11. Gurbel PA, Becker RC, Mann KG, Steinhubl SR, Michelson AD. Platelet function monitoring in patients with coronary artery disease. J Am Coll Cardiol 2007;50:1822-34.

12. MacMullan PA, Peace AJ, Madigan AM, Tedesco AF, Kenny D, McCarthy GM. Platelet hyper-reactivity in active inflammatory arthritis is unique to the adenosine diphosphate pathway: A novel finding and potential therapeutic target. Rheumatology 2010; 49:240-5.

13. Catella-Lawson F, Reilly MP, Kapoor SC, Cucchiara AJ, DeMarco $\mathrm{S}$, Tournier B, et al. Cyclooxygenase inhibitors and the antiplatelet effects of aspirin. N Engl J Med 2001;345:1809-17.

14. Bernlochner I, Steinhubl S, Braun S, Morath T, Jaitner J, Stegherr J, et al. Association between inflammatory biomarkers and platelet aggregation in patients under chronic clopidogrel treatment. Thromb Haemost 2010;104:1193-200.

J Rheumatol 2012;39:210-11; doi:10.3899/jrheum.111356 